\title{
Generalized Helicoidal Surfaces in Euclidean 5-space
}

\author{
Ali Uçum and Makoto Sakaki
}

\begin{abstract}
In this paper, we study generalized helicoidal surfaces in Euclidean 5space. We obtain the necessary and sufficient conditions for generalized helicoidal surfaces in Euclidean 5-space to be minimal, flat or of zero normal curvature tensor, which are ordinary differential equations. We solve those equations and discuss the completeness of the surfaces.
\end{abstract}

\section{Introduction}

In differential geometry, one of the well-known surfaces is helicoidal surfaces. Helicoidal surfaces are a generalization of rotational surfaces. These surfaces are invariant by a subgroup of the group of isometries of the ambient space, called helicoidal group whose elements can be seen as a composition of a translation with a rotation for a given axis. In [4], the authors studied the space of all helicoidal surfaces in Euclidean 3-space which have constant mean curvatures or constant Gaussian curvatures. This space behaves as a circular cylinder, where a given generator corresponds to the rotational surfaces and each parallel corresponds to a periodic family of helicoidal surfaces. In [2], the cases with prescribed mean curvature or Gauss curvature have been studied.

Helicoidal surfaces were studied by many researchers in different spaces. In [6], authors constructed linear Weingarten helicoidal surfaces in Minkowski 3space under the cubic screw motion. In [5], the authors constructed a helicoidal

Key Words: Helicoidal surface, minimal surface, flat surface, normal curvature tensor, complete surface.

2010 Mathematics Subject Classification: Primary 53A05; Secondary 53C42.

Received: 03.03.2021

Accepted: 28.04 .2021 
surface with a light-like axis with prescribed mean curvature or Gauss curvature given by smooth function in Minkowski 3-space and solved an open problem left in [3]. Also, in [7], the authors classify all helicoidal non-degenerate surfaces in Minkowski 3-space with constant mean curvature whose generating curve is the graph of a polynomial or a Lorentzian circle.

Besides, in [1], the authors studied rotational surfaces in higher dimensional Euclidean spaces. They obtained some results related with the curvature properties of these surfaces. Also they give examples of rotational surfaces in Euclidean 5-space.

In this paper, we study generalized helicoidal surfaces in Euclidean 5-space. We obtain the necessary and sufficient conditions for generalized helicoidal surfaces in Euclidean 5-space to be minimal, flat or of zero normal curvature tensor, which are ordinary differential equations. We solve those equations and discuss the completeness of the surfaces.

\section{Preliminaries}

Let $\mathbb{E}^{5}$ be the 5 -dimensional Euclidean space with standard coordinate system $\left\{x_{1}, x_{2}, x_{3}, x_{4}, x_{5}\right\}$ and the metric tensor $g$ has the form

$$
g=\sum_{i=1}^{5}\left(d x_{i}\right)^{2}=\left(d x_{1}\right)^{2}+\left(d x_{2}\right)^{2}+\left(d x_{3}\right)^{2}+\left(d x_{4}\right)^{2}+\left(d x_{5}\right)^{2} .
$$

Let $M$ be a surface immersed in the 5 -dimensional Euclidean space $\mathbb{E}^{5}$. We denote the Levi-Civita connections of $\mathbb{E}^{5}$ and $M$ by $\widetilde{\nabla}$ and $\nabla$, respectively. Let $e_{1}, e_{2}, e_{3}, e_{4}, e_{5}$ be an adapted local orthonormal frame in $\mathbb{E}^{5}$ such that $e_{1}, e_{2}$ are tangent to $M$ and $e_{3}, e_{4}, e_{5}$ are normal to $M$. We know that

$$
\widetilde{\nabla}_{X} Y=\nabla_{X} Y+h(X, Y)
$$

and

$$
\widetilde{\nabla}_{X} \xi=-A_{\xi} X+{ }^{\perp} \nabla_{X} \xi
$$

where $X, Y \in \Gamma(T M)$ and $\xi \in \Gamma\left(T M^{\perp}\right)$. Then $h$ is the second fundamental form, $A_{\xi}$ is the shape operator, and ${ }^{\perp} \nabla$ is the normal connection. We note that

$$
\langle h(X, Y), \xi\rangle=\left\langle A_{\xi} X, Y\right\rangle .
$$

The normal curvature tensor ${ }^{\perp} R$ is defined by

$$
{ }^{\perp} R(X, Y) \xi={ }^{\perp} \nabla_{X}{ }^{\perp} \nabla_{Y} \xi-{ }^{\perp} \nabla_{Y}{ }^{\perp} \nabla_{X} \xi-{ }^{\perp} \nabla_{[X, Y]} \xi,
$$


where $X, Y \in \Gamma(T M)$ and $\xi \in \Gamma\left(T M^{\perp}\right)$. Taking the normal part of the following equation

$$
\widetilde{\nabla}_{X} \widetilde{\nabla}_{Y} \xi-\widetilde{\nabla}_{Y} \widetilde{\nabla}_{X} \xi-\widetilde{\nabla}_{[X, Y]} \xi=0
$$

where $X, Y \in \Gamma(T M)$ and $\xi \in \Gamma\left(T M^{\perp}\right)$, we get the Ricci equation

$$
\left\langle{ }^{\perp} R(X, Y) \xi, \eta\right\rangle=\left\langle A_{\eta} X, A_{\xi} Y\right\rangle-\left\langle A_{\xi} X, A_{\eta} Y\right\rangle
$$

where $\eta \in \Gamma\left(T M^{\perp}\right)$.

We may use the ranges of indices:

$$
1 \leq i, j, k, \ldots \leq 2, \quad 3 \leq r, s, t, \ldots \leq 5, \quad 1 \leq A, B, C, \ldots \leq 5 .
$$

Set

$$
h_{i j}^{s}=\left\langle h\left(e_{i}, e_{j}\right), e_{s}\right\rangle
$$

and

$$
R_{r i j}^{s}=\left\langle{ }^{\perp} R\left(e_{i}, e_{j}\right) e_{r}, e_{s}\right\rangle,
$$

which are the components of the second fundamental form $h$ and the normal curvature tensor ${ }^{\perp} R$, respectively.

By the Ricci equation, the normal curvature tensor satisfies

$$
R_{r i j}^{s}=\left(\left\langle A_{e_{s}} e_{i}, A_{e_{r}} e_{j}\right\rangle-\left\langle A_{e_{r}} e_{i}, A_{e_{s}} e_{j}\right\rangle\right) .
$$

Noting that

$$
A_{e_{s}} e_{i}=\sum_{k} h_{i k}^{s} e_{k}
$$

we obtain

$$
R_{r i j}^{s}=\sum_{k}\left(h_{i k}^{s} h_{j k}^{r}-h_{j k}^{s} h_{i k}^{r}\right) .
$$

Also the mean curvature vector $H$ of $M$ in $\mathbb{E}^{5}$ is defined by

$$
H=\frac{1}{2} \sum_{s}\left(h_{11}^{s}+h_{22}^{s}\right) e_{s} .
$$

A surface $M$ is called minimal if $H=0$ identically.

The Gauss curvature $K$ of $M$ in $\mathbb{E}^{5}$ is given by

$$
K=\sum_{s}\left(h_{11}^{s} h_{22}^{s}-\left(h_{12}^{s}\right)^{2}\right) .
$$

A surface $M$ is called flat if $K=0$ identically. 


\section{Generalized helicoidal surfaces in $\mathbb{E}^{5}$}

In this section, we discuss the geometric properties of a generalized helicoidal surface $M$ in $\mathbb{E}^{5}$ with the parametrization

$$
M: \quad F(t, u)=(\alpha(t) \cos u, \alpha(t) \sin u, \beta(t) \cos u, \beta(t) \sin u, u)
$$

where $(\alpha(t))^{2}+(\beta(t))^{2}>0$ and $\left(\alpha^{\prime}(t)\right)^{2}+\left(\beta^{\prime}(t)\right)^{2}>0$. Then we have

$$
\begin{aligned}
F_{t} & =\left(\alpha^{\prime}(t) \cos u, \alpha^{\prime}(t) \sin u, \beta^{\prime}(t) \cos u, \beta^{\prime}(t) \sin u, 0\right), \\
F_{u} & =(-\alpha(t) \sin u, \alpha(t) \cos u,-\beta(t) \sin u, \beta(t) \cos u, 1)
\end{aligned}
$$

and

$$
\left\langle F_{t}, F_{t}\right\rangle=\left(\alpha^{\prime}(t)\right)^{2}+\left(\beta^{\prime}(t)\right)^{2}, \quad\left\langle F_{t}, F_{u}\right\rangle=0, \quad\left\langle F_{u}, F_{u}\right\rangle=1+\alpha^{2}(t)+\beta^{2}(t) .
$$

Then we can choose the followings:

$$
\begin{aligned}
& e_{1}=\frac{1}{\sqrt{\left(\alpha^{\prime}\right)^{2}+\left(\beta^{\prime}\right)^{2}}} F_{t}=\frac{1}{\sqrt{\left(\alpha^{\prime}\right)^{2}+\left(\beta^{\prime}\right)^{2}}}\left(\alpha^{\prime} \cos u, \alpha^{\prime} \sin u, \beta^{\prime} \cos u, \beta^{\prime} \sin u, 0\right), \\
& e_{2}=\frac{1}{\sqrt{1+\alpha^{2}+\beta^{2}}} F_{u}=\frac{1}{\sqrt{1+\alpha^{2}+\beta^{2}}}(-\alpha \sin u, \alpha \cos u,-\beta \sin u, \beta \cos u, 1), \\
& e_{3}=\frac{1}{\sqrt{\alpha^{2}+\beta^{2}}}(\beta \sin u,-\beta \cos u,-\alpha \sin u, \alpha \cos u, 0), \\
& e_{4}=\frac{1}{\sqrt{\left(\alpha^{\prime}\right)^{2}+\left(\beta^{\prime}\right)^{2}}}\left(-\beta^{\prime} \cos u,-\beta^{\prime} \sin u, \alpha^{\prime} \cos u, \alpha^{\prime} \sin u, 0\right), \\
& e_{5}=\frac{1}{\sqrt{\alpha^{2}+\beta^{2}} \sqrt{1+\alpha^{2}+\beta^{2}}}\left(-\alpha \sin u, \alpha \cos u,-\beta \sin u, \beta \cos u,-\alpha^{2}-\beta^{2}\right) .
\end{aligned}
$$

Here $\left\{e_{1}, e_{2}\right\}$ is an orthonormal frame field on $M$ and $\left\{e_{3}, e_{4}, e_{5}\right\}$ is a normal orthonormal frame field to $M$.

Also we can easily obtain that

$$
\begin{aligned}
\widetilde{\nabla}_{e_{1}} e_{1} & =\frac{\left(\beta^{\prime} \alpha^{\prime \prime}-\alpha^{\prime} \beta^{\prime \prime}\right)}{\left(\left(\alpha^{\prime}\right)^{2}+\left(\beta^{\prime}\right)^{2}\right)^{2}}\left(\beta^{\prime} \cos u, \beta^{\prime} \sin u,-\alpha^{\prime} \cos u,-\alpha^{\prime} \sin u, 0\right), \\
\widetilde{\nabla}_{e_{2}} e_{1} & =\frac{1}{\sqrt{\left(\alpha^{\prime}\right)^{2}+\left(\beta^{\prime}\right)^{2}} \sqrt{1+\alpha^{2}+\beta^{2}}}\left(-\alpha^{\prime} \sin u, \alpha^{\prime} \cos u,-\beta^{\prime} \sin u, \beta^{\prime} \cos u, 0\right), \\
\widetilde{\nabla}_{e_{2}} e_{2} & =\frac{1}{1+\alpha^{2}+\beta^{2}}(-\alpha \cos u,-\alpha \sin u,-\beta \cos u,-\beta \sin u, 0) .
\end{aligned}
$$


The components of the second fundamental form $h$ are given as follows

$$
\begin{gathered}
h_{11}^{4}=\frac{-\beta^{\prime} \alpha^{\prime \prime}+\alpha^{\prime} \beta^{\prime \prime}}{\left(\left(\alpha^{\prime}\right)^{2}+\left(\beta^{\prime}\right)^{2}\right)^{3 / 2}}, \quad h_{12}^{3}=\frac{-\beta \alpha^{\prime}+\alpha \beta^{\prime}}{\sqrt{\left(\alpha^{\prime}\right)^{2}+\left(\beta^{\prime}\right)^{2}} \sqrt{1+\alpha^{2}+\beta^{2}} \sqrt{\alpha^{2}+\beta^{2}}}, \\
h_{12}^{5}=\frac{\alpha \alpha^{\prime}+\beta \beta^{\prime}}{\left(1+\alpha^{2}+\beta^{2}\right) \sqrt{\left(\alpha^{\prime}\right)^{2}+\left(\beta^{\prime}\right)^{2}} \sqrt{\alpha^{2}+\beta^{2}}}, \quad h_{22}^{4}=\frac{-\beta \alpha^{\prime}+\alpha \beta^{\prime}}{\left(1+\alpha^{2}+\beta^{2}\right) \sqrt{\left(\alpha^{\prime}\right)^{2}+\left(\beta^{\prime}\right)^{2}}}, \\
h_{11}^{3}=h_{11}^{5}=h_{12}^{4}=h_{22}^{3}=h_{22}^{5}=0 .
\end{gathered}
$$

Then we get the following theorem and corollary.

Theorem 1. Let $M$ be generalized helicoidal surface parametrized by (3.1). Then the mean curvature vector $H$ of $M$ is given by

$$
H=\frac{1}{2}\left(\frac{-\beta^{\prime} \alpha^{\prime \prime}+\alpha^{\prime} \beta^{\prime \prime}}{\left(\left(\alpha^{\prime}\right)^{2}+\left(\beta^{\prime}\right)^{2}\right)^{3 / 2}}+\frac{-\beta \alpha^{\prime}+\alpha \beta^{\prime}}{\left(1+\alpha^{2}+\beta^{2}\right) \sqrt{\left(\alpha^{\prime}\right)^{2}+\left(\beta^{\prime}\right)^{2}}}\right) e_{4} .
$$

Corollary 1. Let $M$ be generalized helicoidal surface parametrized by (3.1). Then $M$ is minimal if and only if

$$
\frac{\alpha^{\prime} \beta^{\prime \prime}-\beta^{\prime} \alpha^{\prime \prime}}{\left(\alpha^{\prime}\right)^{2}+\left(\beta^{\prime}\right)^{2}}=\frac{\beta \alpha^{\prime}-\alpha \beta^{\prime}}{\left(1+\alpha^{2}+\beta^{2}\right)} .
$$

Let $\beta(t)=t$ in the equation (3.2). Then the minimal surface equation is

$$
\left(\alpha^{2}+t^{2}+1\right) \alpha^{\prime \prime}+\left(t \alpha^{\prime}-\alpha\right)\left(\left(\alpha^{\prime}\right)^{2}+1\right)=0 .
$$

If $\alpha(t)$ is a linear function, that is, $\alpha(t)=p t+q$, then from the above equation, we have $q=0$ and $\alpha(t)=p t$. Then the surface $M$ is a helicoid in a 3dimensional subspace of $\mathbb{E}^{5}$. So, in the following, we will consider the case where $\alpha(t)$ is a nonlinear function.

Multiplying (3.3) by $2 \alpha^{\prime} /\left(\left(\alpha^{\prime}\right)^{2}+1\right)^{2}$, we can get

$$
\left(t^{2} \frac{\left(\alpha^{\prime}\right)^{2}}{\left(\alpha^{\prime}\right)^{2}+1}\right)^{\prime}-\left(\frac{\alpha^{2}+1}{\left(\alpha^{\prime}\right)^{2}+1}\right)^{\prime}=0
$$

Thus we have

$$
t^{2} \frac{\left(\alpha^{\prime}\right)^{2}}{\left(\alpha^{\prime}\right)^{2}+1}-\frac{\alpha^{2}+1}{\left(\alpha^{\prime}\right)^{2}+1}=c_{1}
$$


for a constant $c_{1}$. Then

$$
\alpha^{\prime}= \pm \sqrt{\frac{\alpha^{2}+1+c_{1}}{t^{2}-c_{1}}}
$$

and

$$
\frac{\alpha^{\prime}}{\sqrt{\alpha^{2}+1+c_{1}}}= \pm \frac{1}{\sqrt{t^{2}-c_{1}}} .
$$

Changing $t$ to $-t$ if necessary, we may only consider the $(+)$ case.

(i) When $c_{1}=0$, we have

$$
\frac{\alpha^{\prime}}{\sqrt{\alpha^{2}+1}}=\frac{1}{t}
$$

Integrating it we have

$$
\log \left|\sqrt{\alpha^{2}+1}+\alpha\right|=\log |t|+c_{2}
$$

for a constant $c_{2}$ and

$$
\sqrt{\alpha^{2}+1}+\alpha=c_{3} t
$$

where $c_{3} \neq 0$ is constant. Thus we get

$$
\alpha=\frac{1}{2}\left(c_{3} t-\frac{1}{c_{3} t}\right)
$$

and its graph is a hyperbola. The corresponding surface $M$ is a complete minimal surface.

(ii) When $c_{1} \neq 0$, integrating the equation (3.4), we have

$$
\log \left|\sqrt{\alpha^{2}+1+c_{1}}+\alpha\right|=\log \left|\sqrt{t^{2}-c_{1}}+t\right|+c_{2}
$$

for a constant $c_{2}$, and

$$
\sqrt{\alpha^{2}+1+c_{1}}+\alpha=c_{3}\left(\sqrt{t^{2}-c_{1}}+t\right)
$$

where $c_{3} \neq 0$ is constant. Thus we get

$$
\alpha=\frac{1}{2 c_{1} c_{3}}\left[\left(c_{1} c_{3}^{2}-1-c_{1}\right) t+\left(c_{1} c_{3}^{2}+1+c_{1}\right) \sqrt{t^{2}-c_{1}}\right] .
$$

Since $\alpha(t)$ is not a linear function, we have $c_{1} c_{3}^{2}+1+c_{1} \neq 0$. When $c_{1}<0$, the function $\alpha(t)$ is defined for any $t \in \mathbb{R}$ and its graph is a hyperbola. So we have a complete minimal surface. 
When $c_{1}>0$, we return to the equation (3.4) and rewrite it as

$$
\frac{t^{\prime}}{\sqrt{t^{2}-c_{1}}}=\frac{1}{\sqrt{\alpha^{2}+1+c_{1}}}, \quad t^{\prime}=\frac{d t}{d \alpha} .
$$

Integrating it with respect to $\alpha$, we have

$$
\log \left|\sqrt{t^{2}-c_{1}}+t\right|=\log \left|\sqrt{\alpha^{2}+1+c_{1}}+\alpha\right|+c_{2}
$$

for constant $c_{2}$, and

$$
\sqrt{t^{2}-c_{1}}+t=c_{3}\left(\sqrt{\alpha^{2}+1+c_{1}}+\alpha\right)
$$

where $c_{3} \neq 0$ is constant. Hence

$$
t=\frac{1}{2 c_{3}\left(1+c_{1}\right)}\left[\left(c_{3}^{2}+c_{1} c_{3}^{2}-c_{1}\right) \alpha+\left(c_{3}^{2}+c_{1} c_{3}^{2}+c_{1}\right) \sqrt{\alpha^{2}+1+c_{1}}\right] .
$$

Since $t(\alpha)$ is not a linear function, we have $c_{3}^{2}+c_{1} c_{3}^{2}+c_{1} \neq 0$. The function $t(\alpha)$ is defined for any $\alpha \in \mathbb{R}$ and its graph is hyperbola. So we get a complete minimal surface also in this case.

Theorem 2. The nonlinear solution of the minimal surface equation (3.3) is given by

$$
\begin{gathered}
\alpha=\frac{1}{2}\left(c_{3} t-\frac{1}{c_{3} t}\right), \\
\alpha=\frac{1}{2 c_{1} c_{3}}\left[\left(c_{1} c_{3}^{2}-1-c_{1}\right) t+\left(c_{1} c_{3}^{2}+1+c_{1}\right) \sqrt{t^{2}-c_{1}}\right], \quad\left(c_{1}<0\right)
\end{gathered}
$$

or

$t=\frac{1}{2 c_{3}\left(1+c_{1}\right)}\left[\left(c_{3}^{2}+c_{1} c_{3}^{2}-c_{1}\right) \alpha+\left(c_{3}^{2}+c_{1} c_{3}^{2}+c_{1}\right) \sqrt{\alpha^{2}+1+c_{1}}\right], \quad\left(c_{1}>0\right)$, where $c_{1}$ and $c_{3} \neq 0$ are constants. The corresponding surface $M$ is a complete minimal surface in any case.

In the following theorem, we give the Gauss curvature of the surface (3.1) .

Theorem 3. Let $M$ be generalized helicoidal surface parametrized by (3.1). Then the Gauss curvature $K$ of $M$ is given by

$$
K=\frac{\left(\alpha^{\prime} \beta^{\prime \prime}-\beta^{\prime} \alpha^{\prime \prime}\right)\left(\alpha \beta^{\prime}-\beta \alpha^{\prime}\right)}{\left(\left(\alpha^{\prime}\right)^{2}+\left(\beta^{\prime}\right)^{2}\right)^{2}\left(1+\alpha^{2}+\beta^{2}\right)}-\frac{\left(\alpha \beta^{\prime}-\beta \alpha^{\prime}\right)^{2}\left(1+\alpha^{2}+\beta^{2}\right)+\left(\alpha \alpha^{\prime}+\beta \beta^{\prime}\right)^{2}}{\left(\left(\alpha^{\prime}\right)^{2}+\left(\beta^{\prime}\right)^{2}\right)\left(1+\alpha^{2}+\beta^{2}\right)^{2}\left(\alpha^{2}+\beta^{2}\right)} .
$$


Corollary 2. Let $M$ be generalized helicoidal surface parametrized by (3.1). Then $M$ is flat if and only if

$$
\frac{\left(\alpha^{\prime} \beta^{\prime \prime}-\beta^{\prime} \alpha^{\prime \prime}\right)\left(\alpha \beta^{\prime}-\beta \alpha^{\prime}\right)}{\left(\left(\alpha^{\prime}\right)^{2}+\left(\beta^{\prime}\right)^{2}\right)}=\frac{\left(\alpha \beta^{\prime}-\beta \alpha^{\prime}\right)^{2}\left(1+\alpha^{2}+\beta^{2}\right)+\left(\alpha \alpha^{\prime}+\beta \beta^{\prime}\right)^{2}}{\left(1+\alpha^{2}+\beta^{2}\right)\left(\alpha^{2}+\beta^{2}\right)} .
$$

To study flat surfaces, it is convenient to let

$$
\alpha(t)=P(t) \cos (Q(t)) \quad \text { and } \quad \beta(t)=P(t) \sin (Q(t))
$$

where $P(t)>0$ and $Q(t)$ are nonconstant smooth functions. From the equation (3.5), we have

$$
K=\frac{-\left(P^{\prime}\right)^{4}+P^{4}\left(P^{\prime}\right)^{2}\left(Q^{\prime}\right)^{2}-P^{3}\left(1+P^{2}\right)\left(Q^{\prime}\right)^{2} P^{\prime \prime}+P^{3}\left(1+P^{2}\right) P^{\prime} Q^{\prime} Q^{\prime \prime}}{\left(1+P^{2}\right)^{2}\left(\left(P^{\prime}\right)^{2}+P^{2}\left(Q^{\prime}\right)^{2}\right)^{2}} .
$$

Then the surface $M$ is flat if and only if

$$
-\left(P^{\prime}\right)^{4}+P^{4}\left(P^{\prime}\right)^{2}\left(Q^{\prime}\right)^{2}-P^{3}\left(1+P^{2}\right)\left(Q^{\prime}\right)^{2} P^{\prime \prime}+P^{3}\left(1+P^{2}\right) P^{\prime} Q^{\prime} Q^{\prime \prime}=0 .
$$

Here for $Q(t)=t$, the equation (3.7) is rewritten as

$$
-\left(P^{\prime}\right)^{4}+P^{4}\left(P^{\prime}\right)^{2}-P^{3}\left(1+P^{2}\right) P^{\prime \prime}=0 .
$$

Multiplying the above equation by $2 P P^{\prime}=\left(P^{2}\right)^{\prime}$, we have

$$
-P^{4}\left(1+P^{2}\right)\left(\left(P^{\prime}\right)^{2}\right)^{\prime}+P^{4}\left(P^{2}\right)^{\prime}\left(P^{\prime}\right)^{2}-\left(P^{2}\right)^{\prime}\left(P^{\prime}\right)^{4}=0 .
$$

Dividing by $P^{4}\left(P^{\prime}\right)^{4}$, we get

$$
\left(\frac{1+P^{2}}{\left(P^{\prime}\right)^{2}}\right)^{\prime}+\left(\frac{1}{P^{2}}\right)^{\prime}=0
$$

So

$$
\frac{1+P^{2}}{\left(P^{\prime}\right)^{2}}+\frac{1}{P^{2}}=\frac{1}{c_{1}^{2}}
$$

for a positive constant $c_{1}$. Then

$$
P^{\prime}= \pm c_{1} P \sqrt{\frac{1+P^{2}}{P^{2}-c_{1}^{2}}}
$$


Thus we find

$$
\frac{d t}{d P}= \pm \frac{1}{c_{1} P} \sqrt{\frac{P^{2}-c_{1}^{2}}{1+P^{2}}}
$$

Then

$$
t(P)= \pm \int \frac{1}{c_{1} P} \sqrt{\frac{P^{2}-c_{1}^{2}}{1+P^{2}}} d P .
$$

Let

$$
I:=\int \frac{1}{c_{1} P} \sqrt{\frac{P^{2}-c_{1}^{2}}{1+P^{2}}} d P .
$$

Set

$$
\sqrt{\frac{P^{2}-c_{1}^{2}}{1+P^{2}}}=: s \text {. }
$$

Then

$$
P^{2}=\frac{s^{2}+c_{1}^{2}}{1-s^{2}}
$$

and

$$
P d P=\frac{1+c_{1}^{2}}{\left(1-s^{2}\right)^{2}} s d s .
$$

So we have

$$
\begin{aligned}
I= & \frac{1+c_{1}^{2}}{c_{1}} \int \frac{s^{2}}{\left(1-s^{2}\right)\left(s^{2}+c_{1}^{2}\right)} d s=\frac{1}{2 c_{1}} \int\left(\frac{1}{1+s}+\frac{1}{1-s}-\frac{2 c_{1}^{2}}{s^{2}+c_{1}^{2}}\right) d s \\
= & \frac{1}{2 c_{1}} \log \left(\frac{1+s}{1-s}\right)-\arctan \left(\frac{s}{c_{1}}\right)+c_{2} \\
= & \frac{1}{2 c_{1}} \log \left(\frac{\sqrt{1+P^{2}}+\sqrt{P^{2}-c_{1}^{2}}}{\sqrt{1+P^{2}}-\sqrt{P^{2}-c_{1}^{2}}}\right)-\arctan \left(\frac{1}{c_{1}} \sqrt{\frac{P^{2}-c_{1}^{2}}{1+P^{2}}}\right)+c_{2} \\
= & \frac{1}{c_{1}} \log \left(\sqrt{1+P^{2}}+\sqrt{P^{2}-c_{1}^{2}}\right)-\arctan \left(\frac{1}{c_{1}} \sqrt{\frac{P^{2}-c_{1}^{2}}{1+P^{2}}}\right) \\
& -\frac{1}{2 c_{1}} \log \left(1+c_{1}^{2}\right)+c_{2},
\end{aligned}
$$

where $c_{2}$ is a constant.

Let

$$
\Phi(P):=\frac{1}{c_{1}} \log \left(\sqrt{1+P^{2}}+\sqrt{P^{2}-c_{1}^{2}}\right)-\arctan \left(\frac{1}{c_{1}} \sqrt{\frac{P^{2}-c_{1}^{2}}{1+P^{2}}}\right) .
$$


We denote by $t_{+}(P)$ and $t_{-}(P)$ the solutions of $(3.9)$ in the $(+)$ and $(-)$ cases, respectively. Then

$$
t_{+}(P)=\Phi(P)+c_{3} \quad \text { and } \quad t_{-}(P)=-\Phi(P)+c_{4}
$$

for some constants $c_{3}$ and $c_{4}$. The function $t_{+}(P)$ is an increasing function on $\left(c_{1}, \infty\right)$ and

$$
\lim _{P \rightarrow \infty} t_{+}(P)=\infty, \quad \lim _{P \rightarrow c_{1}^{+}} t_{+}(P)=\frac{1}{2 c_{1}} \log \left(1+c_{1}^{2}\right)+c_{3}, \quad \lim _{P \rightarrow c_{1}^{+}} t_{+}^{\prime}(P)=0 .
$$

Similarly, $t_{-}(P)$ is a decreasing function on $\left(c_{1}, \infty\right)$ and

$\lim _{P \rightarrow \infty} t_{-}(P)=-\infty, \quad \lim _{P \rightarrow c_{1}^{+}} t_{-}(P)=-\frac{1}{2 c_{1}} \log \left(1+c_{1}^{2}\right)+c_{4}, \quad \lim _{P \rightarrow c_{1}^{+}} t_{-}^{\prime}(P)=0$.

We choose $c_{4}$ such that

$$
\frac{1}{2 c_{1}} \log \left(1+c_{1}^{2}\right)+c_{3}=-\frac{1}{2 c_{1}} \log \left(1+c_{1}^{2}\right)+c_{4}
$$

The curves $\left(P \cos \left(t_{+}(P)\right), P \sin \left(t_{+}(P)\right)\right)$ and $\left(P \cos \left(t_{-}(P)\right), P \sin \left(t_{-}(P)\right)\right)$ can be connected continuously, but it is not a regular curve. So the corresponding surface $M$ cannot be extended as a complete flat surface.

Theorem 4. The solution of the flat surface equation (3.8) is given by

$$
t_{+}(P)=\Phi(P)+c_{3} \quad \text { or } \quad t_{-}(P)=-\Phi(P)+c_{4},
$$

where

$$
\Phi(P)=\frac{1}{c_{1}} \log \left(\sqrt{1+P^{2}}+\sqrt{P^{2}-c_{1}^{2}}\right)-\arctan \left(\frac{1}{c_{1}} \sqrt{\frac{P^{2}-c_{1}^{2}}{1+P^{2}}}\right)
$$

and $c_{1}>0, c_{3}, c_{4}$ are constants. The corresponding surface $M$ cannot be extended as a complete flat surface.

In the following theorem, we consider the case where the normal curvature tensor of $M$ is identically zero.

Theorem 5. Let $M$ be generalized helicoidal surface parametrized by (3.1). Then the normal curvature tensor of $M$ is identically zero if and only if

$$
\left(\left(\alpha^{\prime}\right)^{2}+\left(\beta^{\prime}\right)^{2}\right)\left(\beta \alpha^{\prime}-\alpha \beta^{\prime}\right)+\left(\alpha^{\prime} \beta^{\prime \prime}-\beta^{\prime} \alpha^{\prime \prime}\right)\left(1+\alpha^{2}+\beta^{2}\right)=0 .
$$


Proof. We have

$$
\begin{aligned}
& R_{412}^{3}=h_{11}^{3} h_{21}^{4}-h_{21}^{3} h_{11}^{4}+h_{12}^{3} h_{22}^{4}-h_{22}^{3} h_{12}^{4} \\
& =\frac{\left(\beta \alpha^{\prime}-\alpha \beta^{\prime}\right)\left[\left(\left(\alpha^{\prime}\right)^{2}+\left(\beta^{\prime}\right)^{2}\right)\left(\beta \alpha^{\prime}-\alpha \beta^{\prime}\right)+\left(\alpha^{\prime} \beta^{\prime \prime}-\beta^{\prime} \alpha^{\prime \prime}\right)\left(1+\alpha^{2}+\beta^{2}\right)\right]}{\sqrt{\alpha^{2}+\beta^{2}}\left(1+\alpha^{2}+\beta^{2}\right)^{3 / 2}\left(\left(\alpha^{\prime}\right)^{2}+\left(\beta^{\prime}\right)^{2}\right)^{2}}, \\
& R_{512}^{3}=h_{11}^{3} h_{21}^{5}-h_{21}^{3} h_{11}^{5}+h_{12}^{3} h_{22}^{5}-h_{22}^{3} h_{12}^{5}=0, \\
& R_{512}^{4}=h_{11}^{4} h_{21}^{5}-h_{21}^{4} h_{11}^{5}+h_{12}^{4} h_{22}^{5}-h_{22}^{4} h_{12}^{5} \\
& =\frac{\left(\alpha \alpha^{\prime}+\beta \beta^{\prime}\right)\left[\left(\left(\alpha^{\prime}\right)^{2}+\left(\beta^{\prime}\right)^{2}\right)\left(\beta \alpha^{\prime}-\alpha \beta^{\prime}\right)+\left(\alpha^{\prime} \beta^{\prime \prime}-\beta^{\prime} \alpha^{\prime \prime}\right)\left(1+\alpha^{2}+\beta^{2}\right)\right]}{\sqrt{\alpha^{2}+\beta^{2}}\left(1+\alpha^{2}+\beta^{2}\right)^{2}\left(\left(\alpha^{\prime}\right)^{2}+\left(\beta^{\prime}\right)^{2}\right)^{2}} .
\end{aligned}
$$

Thus ${ }^{\perp} R=0$ if and only if

$$
\left(\left(\alpha^{\prime}\right)^{2}+\left(\beta^{\prime}\right)^{2}\right)\left(\beta \alpha^{\prime}-\alpha \beta^{\prime}\right)+\left(\alpha^{\prime} \beta^{\prime \prime}-\beta^{\prime} \alpha^{\prime \prime}\right)\left(1+\alpha^{2}+\beta^{2}\right)=0 .
$$

To study surfaces with zero normal curvature tensor, it is convenient to let

$$
\alpha(t)=P(t) \cos (Q(t)) \quad \text { and } \quad \beta(t)=P(t) \sin (Q(t))
$$

where $P(t)>0$ and $Q(t)$ are nonconstant smooth functions. From the equation $(3.10)$, we have

$$
P Q^{\prime}\left(P^{\prime \prime}\left(1+P^{2}\right)-P\left(Q^{\prime}\right)^{2}\right)-\left(2+P^{2}\right)\left(P^{\prime}\right)^{2} Q^{\prime}-\left(1+P^{2}\right) P P^{\prime} Q^{\prime \prime}=0 .
$$

Here for $Q(t)=t$, the equation (3.11) is rewritten as

$$
P P^{\prime \prime}\left(1+P^{2}\right)-\left(2+P^{2}\right)\left(P^{\prime}\right)^{2}-P^{2}=0 .
$$

Multiplying by $2 P P^{\prime}=\left(P^{2}\right)^{\prime}$, we have

$$
P^{2}\left(1+P^{2}\right)\left(\left(P^{\prime}\right)^{2}\right)^{\prime}-\left(2+P^{2}\right)\left(P^{2}\right)^{\prime}\left(P^{\prime}\right)^{2}-P^{2}\left(P^{2}\right)^{\prime}=0 .
$$

Dividing by $P^{6}$, we get

$$
\left(\frac{1+P^{2}}{P^{4}}\left(P^{\prime}\right)^{2}\right)^{\prime}+\left(\frac{1}{P^{2}}\right)^{\prime}=0
$$


So

$$
\frac{1+P^{2}}{P^{4}}\left(P^{\prime}\right)^{2}+\frac{1}{P^{2}}=\frac{1}{c_{1}^{2}}
$$

for a positive constant $c_{1}$. Then

$$
P^{\prime}= \pm \frac{P}{c_{1}} \sqrt{\frac{P^{2}-c_{1}^{2}}{1+P^{2}}} .
$$

Thus we find

$$
\frac{d t}{d P}= \pm \frac{c_{1}}{P} \sqrt{\frac{1+P^{2}}{P^{2}-c_{1}^{2}}}
$$

Then

$$
t(P)= \pm \int \frac{c_{1}}{P} \sqrt{\frac{1+P^{2}}{P^{2}-c_{1}^{2}}} d P .
$$

Let

$$
I:=\int \frac{c_{1}}{P} \sqrt{\frac{1+P^{2}}{P^{2}-c_{1}^{2}}} d P .
$$

Set

$$
\sqrt{\frac{1+P^{2}}{P^{2}-c_{1}^{2}}}=: s \text {. }
$$

Then

$$
P^{2}=\frac{1+c_{1}^{2} s^{2}}{s^{2}-1}
$$

and

$$
P d P=-\frac{1+c_{1}^{2}}{\left(s^{2}-1\right)^{2}} s d s .
$$

So we have

$$
\begin{aligned}
& I=-c_{1}\left(1+c_{1}^{2}\right) \int \frac{s^{2}}{\left(s^{2}-1\right)\left(1+c_{1}^{2} s^{2}\right)} d s \\
&=\frac{c_{1}}{2} \int\left(\frac{1}{s+1}-\frac{1}{s-1}-\frac{2}{1+c_{1}^{2} s^{2}}\right) d s \\
&=\frac{c_{1}}{2} \log \left(\frac{s+1}{s-1}\right)-\arctan \left(c_{1} s\right)+c_{2} \\
&=\frac{c_{1}}{2} \log \left(\frac{\sqrt{1+P^{2}}+\sqrt{P^{2}-c_{1}^{2}}}{\sqrt{1+P^{2}}-\sqrt{P^{2}-c_{1}^{2}}}\right)-\arctan \left(c_{1} \sqrt{\frac{1+P^{2}}{P^{2}-c_{1}^{2}}}\right)+c_{2} \\
&=c_{1} \log \left(\sqrt{1+P^{2}}+\sqrt{P^{2}-c_{1}^{2}}\right)-\arctan \left(c_{1} \sqrt{\frac{1+P^{2}}{P^{2}-c_{1}^{2}}}\right)-\frac{c_{1}}{2} \log \left(1+c_{1}^{2}\right)+c_{2},
\end{aligned}
$$


where $c_{2}$ is a constant.

Let

$$
\Psi(P):=c_{1} \log \left(\sqrt{1+P^{2}}+\sqrt{P^{2}-c_{1}^{2}}\right)-\arctan \left(c_{1} \sqrt{\frac{1+P^{2}}{P^{2}-c_{1}^{2}}}\right) .
$$

We denote by $t_{+}(P)$ and $t_{-}(P)$ the solutions of $(3.13)$ in the $(+)$ and $(-)$ cases, respectively. Then

$$
t_{+}(P)=\Psi(P)+c_{3} \quad \text { and } \quad t_{-}(P)=-\Psi(P)+c_{4}
$$

for some constants $c_{3}$ and $c_{4}$.

The function $t_{+}(P)$ is an increasing function on $\left(c_{1}, \infty\right)$ and

$\lim _{P \rightarrow \infty} t_{+}(P)=\infty, \quad \lim _{P \rightarrow c_{1}^{+}} t_{+}(P)=\frac{c_{1}}{2} \log \left(1+c_{1}^{2}\right)-\frac{\pi}{2}+c_{3}, \quad \lim _{P \rightarrow c_{1}^{+}} t_{+}^{\prime}(P)=\infty$.

Similarly, $t_{-}(P)$ is a decreasing function on $\left(c_{1}, \infty\right)$ and

$\lim _{P \rightarrow \infty} t_{-}(P)=-\infty, \lim _{P \rightarrow c_{1}^{+}} t_{-}(P)=-\frac{c_{1}}{2} \log \left(1+c_{1}^{2}\right)+\frac{\pi}{2}+c_{4}, \lim _{P \rightarrow c_{1}^{+}} t_{-}^{\prime}(P)=-\infty$.

We choose $c_{4}$ such that

$$
\frac{c_{1}}{2} \log \left(1+c_{1}^{2}\right)-\frac{\pi}{2}+c_{3}=-\frac{c_{1}}{2} \log \left(1+c_{1}^{2}\right)+\frac{\pi}{2}+c_{4}=: t_{0} .
$$

Let $P_{+}(t)$ denote the inverse function of $t_{+}(P)$. It is an increasing function on $\left(t_{0}, \infty\right)$ and

$$
\lim _{t \rightarrow \infty} P_{+}(t)=\infty, \quad \lim _{t \rightarrow t_{0}^{+}} P_{+}(t)=c_{1} \quad \lim _{t \rightarrow t_{0}^{+}} P_{+}^{\prime}(t)=0 .
$$

Let $P_{-}(t)$ denote the inverse function of $t_{-}(P)$. It is a decreasing function on $\left(-\infty, t_{0}\right)$ and

$$
\lim _{t \rightarrow-\infty} P_{-}(t)=\infty, \quad \lim _{t \rightarrow t_{0}^{-}} P_{-}(t)=c_{1} \quad \lim _{t \rightarrow t_{0}^{-}} P_{-}^{\prime}(t)=0 .
$$

Now we define a function $P(t)$ on $\mathbb{R}$ such that $P(t)=P_{+}(t)$ for $t>t_{0}$, $P\left(t_{0}\right)=c_{1}$ and $P(t)=P_{-}(t)$ for $t<t_{0}$. Then $P(t)$ is a $C^{1}$ function on $\mathbb{R}$ such that $P^{\prime}(t)=P_{+}^{\prime}(t)$ for $t>t_{0}, P^{\prime}\left(t_{0}\right)=0$ and $P^{\prime}(t)=P_{-}^{\prime}(t)$ for $t<t_{0}$. For $t \neq t_{0}$, it satisfies

$$
P^{\prime \prime}(t)=\frac{\left(2+P(t)^{2}\right)\left(P^{\prime}(t)\right)^{2}+P(t)^{2}}{P(t)\left(1+P(t)^{2}\right)} .
$$


Then we have

$$
\lim _{t \rightarrow t_{0}} P^{\prime \prime}(t)=\frac{c_{1}}{1+c_{1}^{2}} .
$$

Thus we find that $P(t)$ is a $C^{2}$ function on $\mathbb{R}$, which satisfies the zero normal curvature tensor equation. So the corresponding surface $M$ is a complete surface with zero normal curvature tensor.

Theorem 6. The solution of the zero normal curvature tensor equation (3.12) is given by

$$
t_{+}(P)=\Psi(P)+c_{3} \quad \text { or } \quad t_{-}(P)=-\Psi(P)+c_{4}
$$

where

$$
\Psi(P)=c_{1} \log \left(\sqrt{1+P^{2}}+\sqrt{P^{2}-c_{1}^{2}}\right)-\arctan \left(c_{1} \sqrt{\frac{1+P^{2}}{P^{2}-c_{1}^{2}}}\right)
$$

and $c_{1}>0, c_{3}, c_{4}$ are constants. The corresponding surface $M$ can be extended as a complete surface with zero normal curvature tensor.

\section{References}

[1] K. Arslan, B. Bayram, B. Bulca, D. Kosova and G. Öztürk, Rotational surfaces in higher dimensional Euclidean spaces, Rend. Circ. Mat. Palermo, II. Ser, 67(2018), 59-66.

[2] C. Baikoussis and T. Koufogioros, Helicoidal surfaces with prescribed mean or Gauss curvature, J. Geom. 63 (1998), 25-29.

[3] C.C. Beneki, G. Kaimakamis and B.J. Papantoniou, Helicoidal surfaces in three-dimensional Minkowski space, J. Math. Anal. Appl. 275 (2002) $586-614$.

[4] M. P. Do Carmo and M. Dajczer, Helicoidal surfaces with constant mean curvature, Tôhoku Math. J. 34(3) (1982), 425-435.

[5] F. Ji and Z. H. Hou, A kind of helicoidal surfaces in 3-dimensional Minkowski space, J. Math. Anal. Appl. 304 (2005) 632-643.

[6] F. Ji and Y. Wang, Linear Weingarten helicoidal surfaces in Minkowski 3-space, Differential Geometry-Dynamical Systems, 12 (2010), 95-101.

[7] R. López and E. Demir, Helicoidal surfaces in Minkowski space with constant mean curvature and constant Gauss curvature, Cent. Eur. J. Math, 12(9) (2014), 1349-1361. 
Ali UÇUM,

Kırıkkale University,

Faculty of Sciences and Arts,

Department of Mathematics, Kırıkkale-Turkey.

Email: aliucum05@gmail.com

Makoto SAKAKI

Graduate School of Science and Technology,

Hirosaki University,

Hirosaki 036-8561, Japan.

Email: sakaki@hirosaki-u.ac.jp 